\title{
A daily physical activity programme increased the rate of weight gain and bone mass in preterm very low birth weight infants
}

\author{
Moyer-Mileur LJ, Brunstetter V,McNaught TP, et al. Daily physical activity program increases bone mineralization and \\ growth in preterm very low birth weight infants. Pediatrics 2000 Nov;106:1088-92.

\section{QUESTION: Is a daily physical activity programme effective in increasing body weight and bone mass in preterm very low birthweight infants?}

\section{Design}

Randomised (unclear allocation concealment), blinded (unclear), controlled trial with a mean follow up of 25 days.

\section{Setting}

A newborn intensive care unit in a university hospital in Salt Lake City, Utah, USA.

\section{Patients}

32 healthy preterm infants ( $53 \%$ boys) who had birth weights between 800 and $1600 \mathrm{~g}$ (mean $1271 \mathrm{~g}$ ), gestational age between 26 and 32 weeks (mean 30 wks), appropriate body size, were tolerating enteral feedings at $\geqslant 110 \mathrm{kcal} / \mathrm{kg}$ of body weight/day, and were not on medications other than appropriate vitamin supplements. Follow up was $100 \%$.

\section{Intervention}

After stratification by birth weight and gestational age, infants were allocated to the physical activity group $(n=16)$ or the control group $(n=16)$. Infants in the physical activity group received daily range of motion exercises with gentle compression and extension or flexion against passive resistance to all extremities for 5-10 minutes. Infants in the control group had a daily interactive period of holding and stroking but no range of motion activity. The intervention continued until a body weight of $2.0 \mathrm{~kg}$ was achieved. Infants in both groups had similar nutrient intakes that met the recommended needs for preterm infants.

\section{Main outcome measures}

Rate of change in body weight, forearm bone length, bone area, bone mineral content, lean and fat mass, length, head circumference, and serum and urine concentrations of bone mineral status.

\section{Main results}

Infants in the physical activity group had a greater rate of mean daily weight gain compared with those in the control group (16.3 v $14.6 \mathrm{~g} / \mathrm{kg} / \mathrm{d}, \mathrm{p}=0.02)$ and greater gains in forearm bone length $(4.0 v 3.1 \mathrm{~cm}, \mathrm{p}=0.02)$, bone area $\left(2.0 v 1.0 \mathrm{~cm}^{2}, \mathrm{p}=0.02\right)$, bone mineral content (231 $v 131 \mathrm{mg}, \mathrm{p}=0.05)$, and lean mass (5.3 $v 4.9 \mathrm{~g}$, $\mathrm{p}=0.04)$. The groups did not differ for length, head circumference, fat mass, or serum or urine values.

\section{Conclusion}

In preterm very low birthweight infants, a daily physical activity programme increased the rate of gain in body weight, forearm length, bone area, lean body mass, and bone mineralisation.

\section{COMMENTARY}

In their interesting study, Moyer-Mileur et al explain that premature birth can increase the risk of osteopaenia of prematurity and rickets. To further compound this, they note that hospitalised, preterm infants have limited physical activity, which may increase bone resorption and demineralisation. Their study provides compelling evidence to support a positive correlation between a daily physical activity programme and growth and bone mass in very low birthweight infants. Study findings are consistent with a previous study by Moyer-Mileur in which they used different absorptiometry equipment. ${ }^{1}$

Although the sample size in this study was small, the authors ensured similarity of comparison groups by stratifying by birth weight and gestational age. Also, they were able to retain $100 \%$ of the sample to the end of the study. The study would have been strengthened if it had included long term follow up and if it had examined potential adverse effects of the physical activity, such as bradycardia, apnoea, or desaturation episodes. It is also important to keep in mind that these were healthy premature infants who were not on medications other than vitamin supplements. Study results would not be generalisable to many premature infants in neonatal intensive care units.

This issue of Evidence-Based Nursing includes a summary of a systematic review of developmental care for premature infants. Developmental care includes a broad range of activities designed to reduce infant stress, such as swaddling and decreasing sensory and physical stimulation. Although at first glance, developmental care and physical activity programmes may seem to be mutually exclusive, they need not be; rather, it is a matter of individual tolerance and timing of activities. We do not know how much stress the infants experienced during the physical activity nor do we know the consequences of developmental care on bone mineralisation and growth.

The activity programme was administered by a trained occupational therapist. Further research is required to determine if there are any adverse effects of the programme, to study generalisability to premature infants in neonatal intensive care units who are sicker, and to evaluate whether the programme can be effectively administered by parents.

Pamela Lemons, CNNP, MS Nurse Practitioner, Developmental Pediatrics Department of Pediatrics Indiana University-Purdue University at Indianapolis Indianapolis, Indiana, USA

1 Moyer-Mileur L, Luetkemeier M, Boomer L, et al. Effect of physical activity on bone mineralization in premature infants. J Pediatr 1995;127:620-5. 